1990-01-01

\title{
Characterization of Metal/Carbon Multilayers by Raman Spectroscopy
}

\author{
David D. Allred \\ allred@byu.edu \\ Qi Wang \\ Jesus González-Hernández
}

Follow this and additional works at: https://scholarsarchive.byu.edu/facpub

Part of the Astrophysics and Astronomy Commons, and the Physics Commons

\section{Original Publication Citation}

D.D. Allred, Wang Qi, and J. González-Hernández, "Characterization of Metal/Carbon Multilayers by Raman Spectroscopy," Layered Structures Heteroepitaxy, Superlattices, Strain and Metastability, Materials Research Society Symposium Proceedings Series 16, 6561 (199). [http://journals.cambridge.org/action/ displayAbstract?fromPage=online\&aid=818521\&fulltextType=RA\&fileld=S1946427455879][http://dx.doi.org/ 1.1557/PROC-16-65].

\section{BYU ScholarsArchive Citation}

Allred, David D.; Wang, Qi; and González-Hernández, Jesus, "Characterization of Metal/Carbon Multilayers by Raman Spectroscopy" (1990). Faculty Publications. 1192.

https://scholarsarchive.byu.edu/facpub/1192

This Peer-Reviewed Article is brought to you for free and open access by BYU ScholarsArchive. It has been accepted for inclusion in Faculty Publications by an authorized administrator of BYU ScholarsArchive. For more information, please contact ellen_amatangelo@byu.edu. 


\section{CHARACTERIZATION OF METAL/CARBON MULTILAYERS BY RAMAN SPECTROSCOPY}

David D. Allred, Qi Wang and Jesus Gonzalez-Hernandez*

Center for X-ray Imaging and Department of Physics and Astronomy, Brigham Young University, Provo, UT 84602

*also Centro de Investigacion y Estudios Avanzados del IPN, Mexico D.F., Mexico

\section{ABSTRACT}

Laser Raman spectroscopy has been found to be useful for characterizing amorphous semiconductor multilayers, especially the interfaces of multilayers. Recently, we have extended this technique to the characterization of magnetron sputtered multilayers commonly used as reflectors in soft $\mathrm{x}$-ray optics. Unlike the multilayers previously studied which contained only semiconductors and dielectrics, these are generally semiconductor/metal multilayers. We report here on the Raman characterization of the most common class of multilayers used in soft $\mathrm{x}$-ray optics, those that contain a high density metal like tungsten interspersed with layers of carbon. In all of the metal/carbon multilayers the dominate feature in the Raman spectra is due to a-C. The a-C spectra consists of a broad peak at about $1560 \mathrm{~cm}^{-1}$ (G-peak) and a shoulder at about $1400 \mathrm{~cm}^{-1}$ (D) peak). This can be deconvoluted with Gaussian line shapes to yield two peaks (one at about 1560 to $1570 \mathrm{~cm}^{-1}$ and the other at about 1380 to $1420 \mathrm{~cm}^{-1}$ ). Among the W/C multilayer samples peak positions and relative magnitudes changed little with carbon thickness over the range of 1 to $12 \mathrm{~nm}$. Significant differences are, however, seen as the identity of the metal component is altered or, especially, as the preparations are varied. For example, the intensity ratio of the D-peak to G-peak was much larger for multilayer samples prepared under conditions of good plasma confinement.

\section{INTRODUCTION}

Raman spectroscopy and low angle $\mathrm{x}$-ray diffraction have been shown to be useful techniques for characterizing amorphous semiconductor multilayers, especially the interfaces of multilayers ${ }^{1-3}$. Multilayers have proven to be a convenient way of studying the nanoscale properties of materials, for example, the crystallization and interdiffusion of matter on atomic scales ${ }^{4-7}$. Technologically, periodic bilayer multilayers have proven to be of key importance in the current revolution in $x$-ray imaging ${ }^{8}$. These achieve narrow band reflectance by having an index difference between the two layers in the period and by having numerous periods. The index difference is large if one of the layers has a high electron density and the other a low electron density.

Multilayers previously studied by Raman methods contain only semiconductors and dielectrics and have too low a difference in index in the soft $x$-ray region to be useful for $\mathrm{x}$-ray imaging. On the other hand, the multilayers commonly used for soft $\mathrm{x}$-ray applications are not a priori suitable for Raman analysis. The high electron density materials are refractory metals such as tungsten. The fact that the Raman spectra of metals are usually faint and the fact that metals are quite absorbing mitigate against the use of Raman spectroscopy in the study of $\mathrm{x}$-ray multilayers. However, the low electron density layers which come between each of the high electron density layers of the periodic multilayer are often semiconductors such as amorphous carbon. Thus the common highperformance, soft $\mathrm{x}$-ray multilayers contain a semiconductor component subject to Raman analysis. In addition, since the multilayers serve for very short wavelength radiation, the metal layers are ultrathin, permitting the probing radiation to sample several of the semiconductor layers. In a few cases the interface itself may have a high Raman cross section allowing the possibility that it also may be studied by Raman spectroscopy. Recently, we have shown this to be the case by extending the use of this technique to the characterization of magnetron sputtered $\mathrm{W} / \mathrm{C}$ multilayers commonly used as reflectors in soft $\mathrm{x}$-ray optics ${ }^{9}$.

In the $\mathrm{W} / \mathrm{C}$ multilayers the Raman spectra of amorphous carbon is seen to dominate. This was the first report of the Raman spectra of amorphous carbon in multilayers of any kind and the first reported use of Raman to characterize metal/semiconductor multilayers.

Mat. Res. Soc. Symp. Proc. Vol, 160. 61990 Materials Research Society 
There are two main bands observed. One is related to the graphite $\mathrm{E}_{2 g}$ mode (G-line) at $\sim 1580 \mathrm{~cm}^{-1}$ and the other, which is due to disorder (D-line), is at $\sim 1390 \mathrm{~cm}^{-1} 10,11$. The information from these two lines, such as central frequencies, the intensity ratio and line widths are useful in the study of the amorphous carbon structure. We found that the position of the peaks is relatively constant and the ratio of their magnitudes is rather constant for all of the multilayer samples. The observed Raman data shows that the intensity ratio of the $\mathrm{D}$ to $\mathrm{G}$ peaks is relatively larger for multilayers than those for the thick carbon film and thin carbon film on thick tungsten.

\section{EXPERIMENTAL}

All samples were prepared by DC magnetron sputtering. The multilayer samples were similar to those used as mirrors in soft $\mathrm{x}$-ray optics. They consist of two sputtered materials: carbon and a metal, for example, tungsten, arranged alternately in layers of the thickness $\mathrm{d}_{C}$ and $\mathrm{d}_{\text {metal }}$ respectively so that the periodicity of the structure was $\mathrm{d}=\mathrm{d}_{C}+\mathrm{d}_{\text {metal }}$. The substrates were $\mathrm{Si}$ wafers and both the top and bottom layers were carbon. There were 20 pairs of layers (periods) in each of the W/C samples. In the first set of samples $\mathrm{d}_{W}$ was kept constant, at a nominal thickness of $2 \mathrm{~nm}$ and $\mathrm{d}_{C}$ ranged from 0.5 to $8 \mathrm{~nm}$. A supplementary set of two multilayer samples also with $\mathrm{d} w=2 \mathrm{~nm}$ (nominal) were prepared later under conditions of somewhat less plasma confinement.

Also prepared were several $\mathrm{Ni}-\mathrm{Cu}$ alloy $/ \mathrm{C}$ and $\mathrm{TaC} / \mathrm{C}$ multilayer samples and a 225 $\mathrm{nm}$ thick a-C film. The composition of the $\mathrm{Ni}-\mathrm{Cu}$ alloy was $67 \% \mathrm{Ni}$ and $33 \% \mathrm{Cu}$ and is often termed monel. The $\mathrm{Ni}-\mathrm{Cu}$ alloy/C sample was $(4 / 4 \mathrm{~nm}) \times 30$. This notation means that there are thirty layers each consisting of $4 \mathrm{~nm}$ of monel and $4 \mathrm{~nm}$ of C. Two sets of $\mathrm{TaC} / \mathrm{C}$ samples were examined. The two sets were similar except that the second (see Fig. 2b) was prepared in a mode where the plasma was not strongly confined and substrate bombardment and heating during growth was experienced. A typical TaC/C sample (see Fig. 2) was $(1.5 / 3 \mathrm{~nm}) \times 50$.

The $d$-spacings of the multilayer samples was determined by using low angle $x$ ray diffraction. By comparing the relative intensity ratios of the peaks of the various diffraction orders with those from computer generated diffraction patterns it was possible to estimate the values of $\mathrm{d}_{C}$ and $\mathrm{d}_{W}$ for the samples.

The Raman setup has been shown schematically elsewhere ${ }^{9}$. An Ar ion laser (LEXEL 96) was used as the excitation source for the Raman measurements. The $488 \mathrm{~nm}$ line at a power of about $400 \mathrm{~mW}$ was used in most measurements. However, the Raman spectra of the $225 \mathrm{~nm}$ a-C film was obtained at five lines between 514 and $457 \mathrm{~nm}$. The results of this study are shown in Fig. 1. The laser beam was focused as a line onto the sample surface by a cylindrical lens. The oblique angle of illumination of the surface (about $70^{\circ}$ to the surface normal) maximized the coupling of light into the sample by minimizing front surface reflectance.

The collection lenses, normal to the sample's surface, collected and focused the scattered light on the entrance slit of a SPEX 1877 Triplemate spectrometer. The spectrometer consists of a modified Czerny-Turner zero-dispersion double spectrometer as the filter stage and a modified Czerny-Turner spectrograph. The dispersion element used in the spectrograph stage was a 1800 grooves/mm holographic grating with $0.92 \mathrm{~nm} / \mathrm{mm}$ dispersion. The spectral coverage was set at about $18 \mathrm{~cm}^{-1}$

At the exit slit, a photomultiplier (EMI 7981B) and photon counting electronics were used to convert the light signal into the analog electrical signal which was stored digitally using a HP-216 computer controlling a TransEra-MDAS-7000 data acquisition system. The digital system (via signal averaging) was essential for signal enhancement for samples containing the smallest amounts of carbon. The computer was also used to control the wavelength drive for the spectrometer. The wavelength-dependent intensity response of the whole Raman spectrum collecting system, collection lenses, spectrometer and photomultiplier were calibrated by using a 45 -W tungsten-halogen lamp (Optronic Laboratories Standards).

\section{RESULTS AND DATA FITTING}

Figures 2-4 respectively show the Raman spectra of sputtered $\mathrm{TaC} / \mathrm{C}$ $((1.5 / 3 \mathrm{~nm}) \times 50), W / \mathrm{C}$ and monel $/ \mathrm{C}((4 / 4 \mathrm{~nm}) \times 30)$ multilayers over the $800-1800 \mathrm{~cm}^{-1}$ 


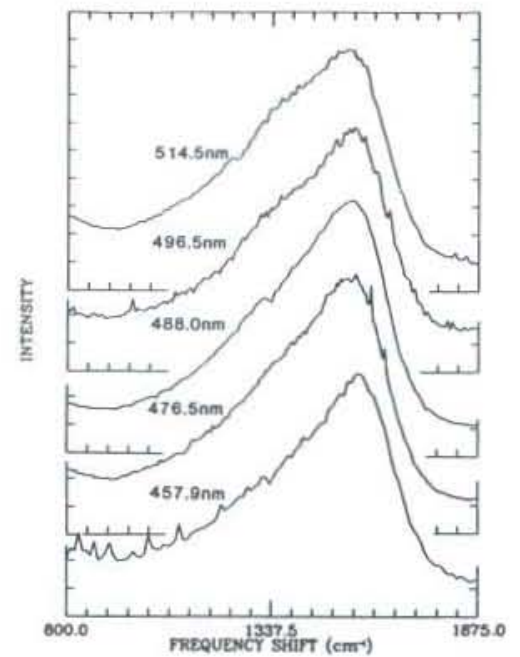

Fig. 1. Raman spectra of a 225 nm sputtered a-C layer. Note the shoulder at about $1350 \mathrm{~cm}^{-1}$ becomes relatively less important at shorter probe wavelengths. less plasma confinement.

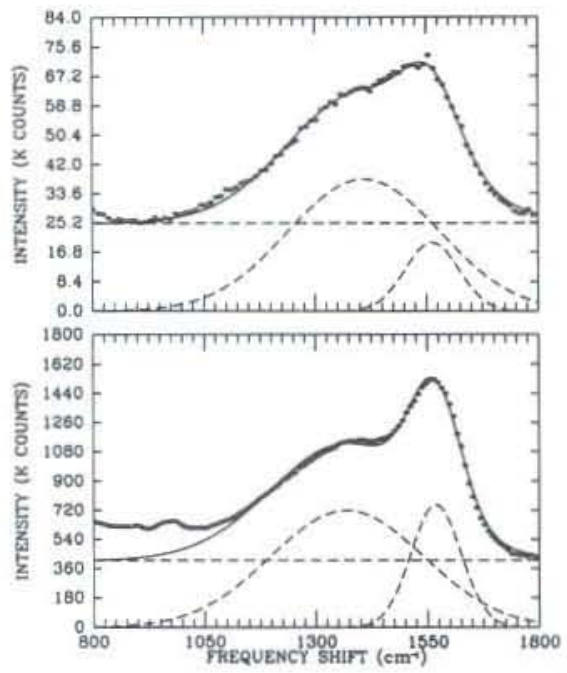

Fig. 2. Raman spectrum of two $\mathrm{TaC} / \mathrm{C}$ multilayers fitted by two Gaussians. From left to right: "graphite"(G), "disordered" (D) and a baseline, a) $(1.5 / 3 \mathrm{~nm}) \times 50 \mathrm{~b})(1.5 / 2.3 \mathrm{~nm}) \times 30$

region of frequency shift, which is the domain where the dominant Raman signal is ob. served. Similarity of the spectra with that of the $200 \mathrm{~nm}$ a-C film (fig. 1) demonstrates that the signal in the multilayers is due to a-C. The metal layers in the multilayer act primarily as an attenuator to the probe beam and the Raman signal from the carbon layers underneath. Thus the intensity of the signal increases monotonically with increasing C thickness in fig. 3.
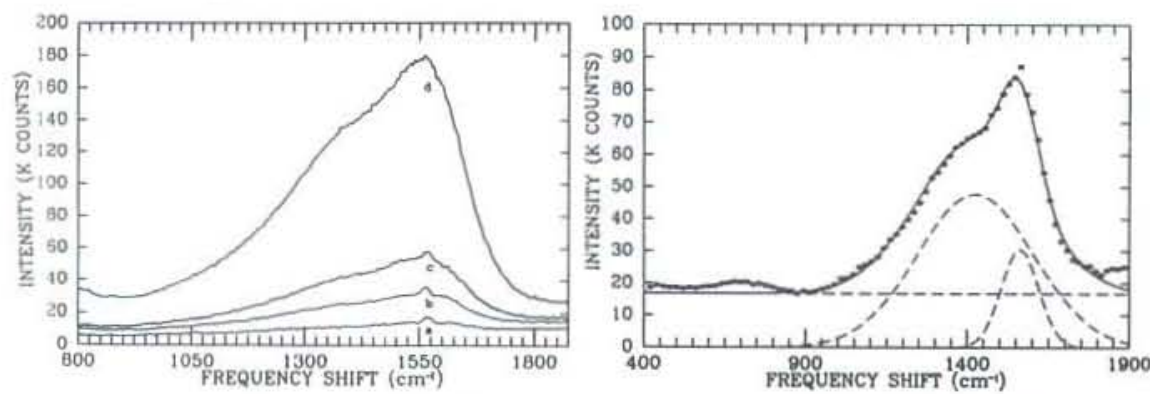

Fig. 3. Raman spectra of $\mathrm{W} / \mathrm{C}$ multilayers. Fig. 4. Raman spectrum of a monel/C multiThe multilayers consist of 20 periods. The $\mathrm{d} w$ in each layer is nominally a constant $2 \mathrm{~nm} ; \mathrm{d}_{C}$ in each layer is $1,2,4$ and $8 \mathrm{~nm}$ layer fitted by two Gaussians. "graphite" (G), "disordered" (D) and a baseline. Note for the curves labeled a to $d$.

The Raman spectra, for all the various samples, between 900 and $1800 \mathrm{~cm}^{-1}$ is dominated by two broad features, a peak, which is associated with the graphite G-line at 
about $1580 \mathrm{~cm}^{-1}$ and a shoulder, associated with the graphite D-line at about $1390 \mathrm{~cm}^{-1}$. The appearance of the Raman spectra depends on the relative magnitude of the two features and to a lesser extent on any changes in relative positions and widths. To better understand the relationship between the features, the observed Raman spectrum for carbon films in this region is frequently treated as the envelope of a D-peak and a Gpeak and is deconvoluted as such ${ }^{9,12,13}$. The increase in the intensity of the peak relative to the shoulder as the excitation wavelength decreases, in Fig. 1, demonstrates that the features can be treated independently. In our curve fitting procedure the envelope was decomposed into two Gaussian shaped lines and a baseline. Only Gaussians adequately fit the high frequency shift side of the $\mathrm{G}$ peak. Various fits to the data are possible depending on how the base line is chosen. This allowed us to estimate the accuracy to which the parameters can be fitted to the data. In Fig. 2 and 4 we show with dashed lines the deconvolution of the data. The fitting is good except sometimes around $1400 \mathrm{~cm}^{-1}$ The deviation below $1000 \mathrm{~cm}^{-1}$ is due to other carbon Raman peaks at lower frequencies and they are not considered any further in this study.

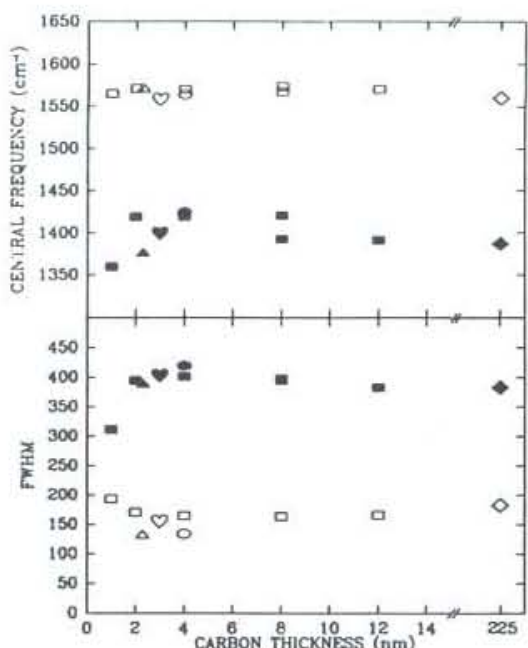

Fig. 5. Central frequency and FWHM of the D and G-peaks for metal/C multilayers as a function of $\mathrm{d}_{C}$. Empty symbols for $\mathrm{G}$ peak; special characters defined in Fig 6.

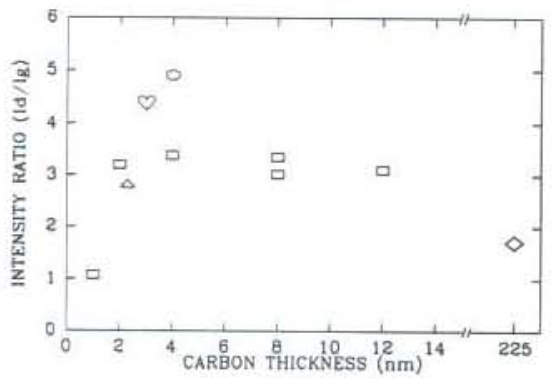

Fig. 6. $\mathrm{I}_{D} / \mathrm{I}_{G}$ for metal $/ \mathrm{C}$ multilayers as a function of $\mathrm{d}_{C}$. $\square$ for $\mathrm{W} / \mathrm{C}, \Delta$ for $\mathrm{TaC} / \mathrm{C}$ $(1.5 / 2.3) \times 30,0$ for $\mathrm{TaC} / \mathrm{C}(1.5 / 3 . \mathrm{nm}) \times 50$, $\mathrm{O}$ for monel/C $(4 / 4 \mathrm{~nm}) \times 30$ and $\bullet$ for 225 $\mathrm{nm}$ a-C film.

Fig. $5 \mathrm{a}$ and $\mathrm{b}$ show, respectively, for the multilayer and thick film samples whose spectra are given in fig. 1-4, the change in the $G$ and $D$ peak central frequencies, the full width half maximum (FWHM) of the peaks as a function of $\mathrm{d}_{C}$. Fig. 6 gives the relationship between the intensity ratio of D-peak to $\mathrm{G}$-peak and $\mathrm{d}_{C}$. This ratio is relatively sensitive to fitting for samples which contained the least amount of carbon, namely those with $\mathrm{d}_{C}$ equal to $0.5 \mathrm{~nm}$ and $1.0 \mathrm{~nm}$. Similarly in fig. 5 , the error bars are, generally, larger for samples which produce a weak Raman spectrum such as the $2 \mathrm{~nm}$ $\mathrm{W} / 1 \mathrm{~nm} \mathrm{C}$ multilayer. However, for samples containing a-C layers thicker than $1 \mathrm{~nm}$ the position of the G-line could be obtained to good accuracy $\pm 3 \mathrm{~cm}^{-1}$. The position of the D-peak central frequency is determined with less precision than that of G-peak-, that is, to about $\pm 10 \mathrm{~cm}^{-1}$.

Among the various samples the parameters for the W/C multilayers prepared with good plasma confinement most closely resemble one another. Beyond 1.0nm within the accuracy of the fitting the ratio of $\mathrm{I}_{D} / \mathrm{I}_{G}$ is a constant 3.3. Similarly, as the thickness of 
$\mathrm{d}_{C}$ increases from about $1 \mathrm{~nm}$ to $8 \mathrm{~nm}$ neither the $\mathrm{G}$ or $\mathrm{D}$ peak central frequency shifts significantly. Similarly the FWHM do not show any trend beyond an a-C thickness of $2 \mathrm{~nm}$ for $\mathrm{W} / \mathrm{C}$ multilayers. There are two values given for $\mathrm{W} / \mathrm{C}$ of $8 \mathrm{~nm}$. The lower of these and the point at $12 \mathrm{~nm}$ carbon thickness correspond to samples prepared with a smaller magnetic field. The main difference between these and those prepared with more confinement is the decrease, by about $30 \mathrm{~cm}^{-1}$, in the position of the D peak.

\section{DISCUSSION}

The most marked difference in Raman spectra between various samples is the relatively high magnitude of the shoulder associated with D feature at about $1390 \mathrm{~cm}^{-1}$ in the multilayer samples. It has frequently been found to be the case for elemental semiconductors that the Raman spectra of the amorphous form of the semiconductor is a broadened and shifted form of the polycrystalline form of the material. The Raman spectrum of the crystal can be useful as a guide to the identification of features and trends in the amorphous. Mierocrystalline graphite samples show peaks at about. 1580 and $1390 \mathrm{~cm}^{-1}$, similar to the amorphous samples, albeit much narrower. The origin of the peak at $1580 \mathrm{~cm}^{-1}$ is associated with the mode in graphite (thus the name G-peak) while the feature at approximately $1390 \mathrm{~cm}^{-1}$ in microcrystalline graphite samples is as. sociated with disorder in the samples (thus the name D-peak for this feature). In graphite the magnitude of the D-line has been correlated with the size of particles; following the procedure of Tuinstra et al.the intensity ratios $I_{D} / I_{G}$ is often used to estimate the cluster size $\mathrm{L}_{a} \cdot{ }^{10,13}$ This procedure is not justifiable for amorphous carbon. It is generally thought that the larger the magnitude of the D peak the more the disorder in the film.

Comparisons between the spectra of samples from figures 1-4 and the summary of fitting parameters relative to the various samples in fig. 5-6 show that while the spectra of the multilayer samples resemble one another there can be significant differences. Differences in preparation conditions related to the sputter sources appear to be a major source of variations. There are also significant variations which occur when one metal is replaced by another in the multilayers.

The spectra of carbon in samples from the W/C material system more closely resemble one another than they resemble spectra from other multilayers systems and thicker single layer films of amorphous carbon. This indicates that the structure of the amorphous carbon in W/C multilayers is reasonably constant as a function of thickness in ultrathin a-C films in $\mathrm{W} / \mathrm{C}$ multilayers and further suggests that there is probably no significant Raman active "interfacial" layer in these samples. Since the absorption coefficient of tungsten is much greater than that of carbon the depth which the laser light can penetrate is mainly decided by the thickness of the tungsten. In the set of W/C multilayers the $\mathrm{W}$ thickness is constant so for all $\mathrm{W} / \mathrm{C}$ samples approximately the same number of carbon films and interfaces is sampled. The fact that within a given set of samples the spectra can be so consistent establishes that good reproducibility is possible given the same material system and process parameters. On the other hand, variations from the canonical W/C spectra is evidence that there has been a change in the structure of the carbon.

That changing process parameters may make a difference in the structure of the carbon film is indicated by slight variations in the spectra of the two W/C samples sputtered after the magnetic field in the sputter guns had been altered slightly relative to those sputtered earlier, the lower of the two points at carbon thickness $8 \mathrm{~nm}$ and the point at $12 \mathrm{~nm}$. An even more pronounced variation in the Raman spectra, which can also be traced to variations in deposition conditions, is seen in the two samples of $\mathrm{TaC} / \mathrm{C}$. Of the two, the first spectrum most closely resembles the spectra from the W/C and other multilayers. The primary difference was in preparation. The first $\mathrm{TaC} / \mathrm{C}$ multilayer, like the W/C and monel/C multilayers, was prepared with Sloan magnetron sputter guns configured to provide adequate plasma confinement. The second, which was prepared without a magnetic field of adequately strength to keep the plasma from bombarding the substrates, shows a narrowing of the G and D peaks. Samples sputtered using the low confinement were observed to pass through the plasma and in addition to being bombarded by the plasma were found to be heated by it. Other work has shown similar changes in the Raman spectra when multilayer samples are annealed ${ }^{12}$. 
If process conditions made the only difference in structure then all of the multilayers prepared with the same process conditions should have the same Raman spectra to within the accuracy of the measurement. It can be observed that, in fact, the spectra depends on the identity of the metal in the multilayer. This may be indicative of an interfacial effect. Such an effect has been inferred for isolated ultrathin $\mathrm{C}$ films which show rather different spectra (smaller intensity ratio $\mathrm{I}_{D} / \mathrm{I}_{G}$ ) than observed for the W/C multilayers ${ }^{9}$. The tungsten in multilayer samples is often amorphous whereas, in single layer pair samples, it may be polycrystalline. The carbon in multilayer samples might have grown on $\mathrm{a}-\mathrm{W}$ while in W/C layer pair samples it might have grown on $c$-W. Details of the structure of the metal carbon interface, then, could account for the differences in the ratio of the $I_{D} / I_{G}$ and/or peak positions and widths for the multilayers of this study as well.

\section{CONCLUSION}

Raman spectroscopy was used to examine metal/C multilayer soft $x$-ray reflectors. In all there are two main features, a peak at about $1560 \mathrm{~cm}^{-1}$ and shoulder at about $1400 \mathrm{~cm}^{-1}$. Both are due to amorphous carbon. Deconvoluted with Gaussians they yield two peaks, one at about $1400 \mathrm{~cm}^{-1}$ (the D-peak) and one at $1570 \mathrm{~cm}^{-1}$ (the Gpeak). From the amorphous carbon Raman spectrum we estimated that the carbon in multilayered samples may be more disordered than the carbon in single layer pairs and $200 \mathrm{~nm}$ thick sputtered samples. Changing the identity of the metal component of the multilayer or preparation conditions can produce differences in the Raman spectra. This may be evidence of an interfacial effect. The structure of ultrathin a-C in multilayers is influenced by the identity of the layer on which it is deposited.

\section{ACKNOWLEDGEMENTS}

Samples were prepared by Robert Nelson, Kevin Gray and Eric Gardner under the direction of Bryan Peterson and Larry Knight of Brigham Young University. We gratefully acknowledge their assistance. This work was partially supported by the State of Utah Center for X-ray Imaging.

\section{REFERENCES}

1 David D. Allred, J. Gonzalez-Hernandez, O.V. Nguyen, D. Martin and D. Pawlik, J. Mater. Res. 1(3), 468 (1986).

2 P.D. Persans, A.F. Ruppert, B. Abeles and T. Tiedje, Phys. Rev, B32, 5588 (1985).

${ }^{3}$ B. Abeles and T. Tiedje, Phys. Rev, Lett. 51, 2003 (1983).

4 O. J. Petersen, J. M. Thorne, and L. V. Knight, in Science with Soft X-Rays, edited by F. T. Himpsel and Roger W. Klaffly, (SPIE Proc. 447, Bellingham, WA, 1984) pp. 27-32.

5 A. L. Greer and F. Spaepan, in Synthetic Modulated Structures, edited by L. Chang and B. C. Giessen (Academic, New York, 1985) p. 419.

${ }^{6}$ J. Gonzalez-Hernandez, D. D. Allred, and O. V. Nguyen, in Interfaces, Superlattices, and Thin Films, edited by J. D. Dow and I. K. Schuller, (Material Research Society Proc. 77, Pittsburgh, PA, 1987) pp. 665-670.

${ }^{7}$ L. V. Knight, J. M. Thorne, A. Toor, and T. W. Barbee, Jr., Revue Phys. Appl. 23, 1631 (1988) and ref. cited.

${ }^{8}$ N.M. Ceglio, J. X-ray Sci. and Technol. 1, 7 (1989).

9 Wang Qi, D. D. Allred, L. V. Knight and J. Gonzalez-Hernandez, in X-Ray/EUV Optics for Astronomy and Microscopy, edited by Richard B. Hoover, (SPIE Proc. 1160 , Bellingham, WA, 1989) pp. 229-234.

10 F. Tuinstra and J.L. Koenig, J. Chem. Phys. 53, 1127 (1970).

11 Raphael Tsu, Jesus Gonzalez H., Isaac Hernandez C. and Carlos A. Luengo, Solid State Commun. 24, 809 (1977).

12 Hsiao-chu Tsai and D.B. Bogy, J. Vac. Sci. Technol. A(6), 2307 (1988).

${ }^{13}$ M. Yoshikawa, G.Katagir, H. Ishida and A. Ishitani, Solid State Commun. 66, 1177 (1988). 